\title{
The Complexity of Number Theory
}

\author{
Frank Vega \\ Joysonic, Uzun Mirkova 5, Belgrade, 11000, Serbia \\ vega.frank@gmail.com
}

\begin{abstract}
The Goldbach's conjecture has been described as the most difficult problem in the history of Mathematics. This conjecture states that every even integer greater than 2 can be written as the sum of two primes. This is known as the strong Goldbach's conjecture. The conjecture that all odd numbers greater than 7 are the sum of three odd primes is known today as the weak Goldbach conjecture. A major complexity class is $\operatorname{NSPACE}(\mathrm{S}(\mathrm{n}))$ for some $\mathrm{S}(\mathrm{n})$. We show if the weak Goldbach's conjecture is true, then the problem PRIMES is not in NSPACE(S(n)) for all $\mathrm{S}(\mathrm{n})=\mathrm{o}(\log \mathrm{n})$. However, if PRIMES is not in $\operatorname{NSPACE}(\mathrm{S}(\mathrm{n}))$ for all $\mathrm{S}(\mathrm{n})=\mathrm{o}(\log \mathrm{n})$, then the strong Goldbach's conjecture is true or this has an infinite number of counterexamples. Since Harald Helfgott proved that the weak Goldbach's conjecture is true, then the strong Goldbach's conjecture is true or this has an infinite number of counterexamples, where the case of infinite number of counterexamples statistically seems to be unlikely. In addition, if PRIMES is not in $\operatorname{NSPACE}(\mathrm{S}(\mathrm{n}))$ for all $\mathrm{S}(\mathrm{n})=\mathrm{o}(\log \mathrm{n})$, then the Beal's conjecture is true. Since the Beal's conjecture is a generalization of Fermat's Last Theorem, then this is also a simple and short proof for that Theorem.
\end{abstract}

2012 ACM Subject Classification Theory of computation $\rightarrow$ Complexity classes; Theory of computation $\rightarrow$ Regular languages; Theory of computation $\rightarrow$ Problems, reductions and completeness; Mathematics of computing $\rightarrow$ Number-theoretic computations

Keywords and phrases complexity classes, regular languages, number theory, conjecture, primes

\section{Introduction}

\subsection{Goldbach's conjecture}

Number theory is a branch of pure mathematics devoted primarily to the study of the integers and integer-valued functions [26]. Goldbach's conjecture is one of the most important and unsolved problems in number theory [13]. Nowadays, it is one of the open problems of Hilbert and Landau [13]. Goldbach's original conjecture, written on 7 June 1742 in a letter to Leonhard Euler, states: "... at least it seems that every number that is greater than 2 is the sum of three primes" [10]. This is known as the ternary Goldbach conjecture. We call a prime as a natural number that is greater than 1 and has exactly two divisors, 1 and the number itself [29]. However, the mathematician Christian Goldbach considered 1 as a prime number. Euler replied in a letter dated 30 June 1742 the following statement: "Every even integer greater than 2 can be written as the sum of two primes" [10]. This is known as the strong Goldbach conjecture.

Using Vinogradov's method [28], it has been showed that almost all even numbers can be written as the sum of two primes. In 1973, Chen showed that every sufficiently large even number can be written as the sum of some prime number and a semi-prime [6]. The strong Goldbach conjecture implies the conjecture that all odd numbers greater than 7 are the sum of three odd primes, which is known today as the weak Goldbach conjecture [10]. In 2012 and 2013, Peruvian mathematician Harald Helfgott published a pair of papers claiming to improve major and minor arc estimates sufficiently to unconditionally prove the weak Goldbach conjecture [15], [16]. In this work, we prove the strong Goldbach's conjecture is true or this has an infinite number of counterexamples. 


\subsection{Beal's conjecture}

Fermat's Last Theorem was first conjectured by Pierre de Fermat in 1637, famously in the margin of a copy of Arithmetica where he claimed he had a proof that was too large to fit in the margin [29]. This theorem states that no three positive integers $a, b$, and $c$ can satisfy the equation $a^{n}+b^{n}=c^{n}$ for any integer value of $n$ greater than two [29]. It is not known whether Fermat found a valid proof or not [29]. His proof of one case $(n=4)$ by infinite descent has survived [29]. After many intents, the proof of Fermat's Last Theorem for every integer $n>2$ was finally accomplished, after 358 years, by Andrew Wiles in 1995 [30]. However, the Andrew's proof seems to be quite different to the simple and unknown proof that Fermat claimed.

On the other hand, there is a similar and unsolved conjecture called the Beal's conjecture [17]. This conjecture states if $A^{x}+B^{y}=C^{z}$, where $A, B, C, x, y$ and $z$ are positive integers and $x, y$ and $z$ are all greater than 2, then $A, B$ and $C$ must have a common prime factor [29]. Fermat's Last Theorem can be seen as a special case of the Beal's conjecture restricted to $x=y=z$. Billionaire banker Andrew Beal claims to have discovered this conjecture in 1993 while investigating generalizations of Fermat's Last Theorem [17]. This conjecture has occasionally been referred to as a generalized Fermat equation [4] and the Mauldin or Tijdeman-Zagier conjecture [11].

Beal offered a prize of US $\$ 1,000,000$ to the first person who tries to resolve it [29]. For example, the solution $3^{3}+6^{3}=3^{5}$ has bases with a common factor of 3 , and the solution $7^{6}+7^{7}=98^{3}$ has bases with a common factor of 7 . There are some particular cases which have been proved for this conjecture [8], [22], [25], [5]. There are considerable advances on this topic [19], [9]. We contribute on this subject showing the Beal's conjecture is true.

\section{Background Theory}

In 1936, Turing developed his theoretical computational model [27]. The deterministic and nondeterministic Turing machines have become in two of the most important definitions related to this theoretical model for computation [27]. A deterministic Turing machine has only one next action for each step defined in its program or transition function [27]. A nondeterministic Turing machine could contain more than one action defined for each step of its program, where this one is no longer a function, but a relation [27].

Let $\Sigma$ be a finite alphabet with at least two elements, and let $\Sigma^{*}$ be the set of finite strings over $\Sigma$ [3]. A Turing machine $M$ has an associated input alphabet $\Sigma$ [3]. For each string $w$ in $\Sigma^{*}$ there is a computation associated with $M$ on input $w[3]$. We say that $M$ accepts $w$ if this computation terminates in the accepting state, that is $M(w)=$ "yes" [3]. Note that $M$ fails to accept $w$ either if this computation ends in the rejecting state, that is $M(w)=$ " $n o$ ", or if the computation fails to terminate, or the computation ends in the halting state with some output, that is $M(w)=y$ (when $M$ outputs the string $y$ on the input $w)[3]$.

Another relevant advance in the last century has been the definition of a complexity class. A language over an alphabet is any set of strings made up of symbols from that alphabet [7]. A complexity class is a set of problems, which are represented as a language, grouped by measures such as the running time, memory, etc [7]. The language accepted by a Turing machine $M$, denoted $L(M)$, has an associated alphabet $\Sigma$ and is defined by:

$$
L(M)=\left\{w \in \Sigma^{*}: M(w)=" y e s "\right\} .
$$

Moreover, $L(M)$ is decided by $M$, when $w \notin L(M)$ if and only if $M(w)=$ " $n o "$ [7]. A 
logarithmic space Turing machine has a read-only input tape, a write-only output tape, and $\mathrm{read} /$ write work tapes [27]. The work tapes may contain at most $O(\log n)$ symbols [27]. We use $o$-notation to denote an upper bound that is not asymptotically tight. We formally define $o(g(n))$ as the set

$$
\begin{aligned}
& o(g(n))=\{f(n): \text { for any positive constant } c>0, \text { there exists a constant } \\
& \left.n_{0}>0 \text { such that } 0 \leq f(n)<c \times g(n) \text { for all } n \geq n_{0}\right\} .
\end{aligned}
$$

For example, $2 \times n=o\left(n^{2}\right)$, but $2 \times n^{2} \neq o\left(n^{2}\right)[7]$.

In theoretical computer science and formal language theory, a regular language is a formal language that can be expressed using a regular expression [2]. The complexity class that contains all the regular languages is $R E G$. The complexity class $N S P A C E(f(n))$ is the set of decision problems that can be solved by a nondeterministic Turing machine $M$, using space $f(n)$, where $n$ is the length of the input [18].

\section{Results}

\subsection{Goldbach's conjecture}

- Definition 1. We define the weak Goldbach's language $L_{W G}$ as follows:

$$
L_{W G}=\left\{1^{2 \times n+1} 0^{p} 0^{q} 0^{r}: n \in \mathbb{N} \wedge n \geq 4 \wedge p, q \text { and } r \text { are odd primes } \wedge 2 \times n+1=p+q+r\right\} .
$$

We define the strong Goldbach's language $L_{G}$ as follows:

$$
L_{G}=\left\{1^{2 \times n} 0^{p} 0^{q}: n \in \mathbb{N} \wedge n \geq 3 \wedge p \text { and } q \text { are odd primes } \wedge 2 \times n=p+q\right\} .
$$

- Theorem 2. If the weak Goldbach's conjecture is true, then the weak Goldbach's language $L_{W G}$ is non-regular. Moreover, if the strong Goldbach's conjecture is true, then the strong Goldbach's language $L_{G}$ is non-regular.

Proof. If the weak Goldbach's conjecture is true, then the weak Goldbach's language $L_{W G}$ is equal to the another language $L^{\prime}$ defined as follows:

$$
L^{\prime}=\left\{1^{2 \times n+1} 0^{2 \times n+1}: n \in \mathbb{N} \wedge n \geq 4\right\} .
$$

We can easily prove that $L^{\prime}$ is non-regular using the Pumping lemma for regular languages [23]. Moreover, if the strong Goldbach's conjecture is true, then the strong Goldbach's language $L_{G}$ is equal to the another language $L^{\prime \prime}$ defined as follows:

$$
L^{\prime \prime}=\left\{1^{2 \times n} 0^{2 \times n}: n \in \mathbb{N} \wedge n \geq 3\right\} .
$$

We can easily prove that $L^{\prime \prime}$ is non-regular using the Pumping lemma for regular languages as well [23].

- Definition 3. We define the weak verification Goldbach's language $L_{W V G}$ as follows:

$$
L_{W V G}=\left\{(2 \times n+1, p, q, r) \text { : such that } 1^{2 \times n+1} 0^{p} 0^{q} 0^{r} \in L_{W G}\right\} .
$$

We define the strong verification Goldbach's language $L_{V G}$ as follows:

$$
L_{V G}=\left\{(2 \times n, p, q) \text { : such that } 1^{2 \times n} 0^{p} 0^{q} \in L_{G}\right\} .
$$


- Definition 4. We define the weak Goldbach's language with separator $L_{W S G}$ as follows:

$$
L_{W S G}=\left\{0^{2 \times n+1} \# 0^{p} \# 0^{q} \# 0^{r}: \text { such that } 1^{2 \times n+1} 0^{p} 0^{q} 0^{r} \in L_{W G}\right\}
$$

and we define the strong Goldbach's language with separator $L_{S G}$ as follows:

$$
L_{S G}=\left\{0^{2 \times n} \# 0^{p} \# 0^{q}: \text { such that } 1^{2 \times n} 0^{p} 0^{q} \in L_{G}\right\}
$$

where \# is the blank symbol.

- Lemma 5. The weak Goldbach's language with separator $L_{W S G}$ is the unary representation of the weak verification Goldbach's language $L_{W V G}$. The strong Goldbach's language with separator $L_{S G}$ is the unary representation of the strong verification Goldbach's language $L_{V G}$.

Proof. This is trivially true from the definition of these languages.

- Theorem 6. If $L_{W V G} \in N S P A C E(S(n))$ for some $S(n)=o(\log n)$, then $L_{W G} \in R E G$.

Proof. In case of $L_{W V G} \in N S P A C E(S(n))$ for some $S(n)=o(\log n)$, then there is a nondeterministic Turing machine which decides $L_{W S G}$ that uses space that is smaller than $c \times \log \log n$ for all $c>0$, because of $L_{W S G}$ is the unary version of $L_{W V G}$ due to Lemma 5 [12]. Certainly, the standard space translation between the unary and binary languages actually works for nondeterministic machines with small space [12]. This means that if some language belongs to $N S P A C E(S(n))$, then the unary version of that language belongs to $N S P A C E(S(\log n))$ [12]. In this way, we obtain that $L_{W S G} \in R E G$ because of $R E G=N S P A C E(o(\log \log n))$ [18]. In addition, we can reduce in a nondeterministic constant space the language $L_{W G}$ to $L_{W S G}$ just nondeterministically inserting the blank symbol \# within two arbitrary positions between the 0's on the input. Moreover, this nondeterminism reduction inserts the blank symbol \# between the 1's and 0's and converts the 1's to 0's from the original input of $L_{W G}$ just generating the final output to $L_{W S G}$. Consequently, we prove $L_{W G} \in R E G$ under the assumption that $L_{W V G} \in N S P A C E(S(n))$ for some $S(n)=o(\log n)$, since $R E G$ is also the complexity class of languages decided by nondeterministic Turing machines in constant space [24].

- Theorem 7. $L_{W V G} \notin N S P A C E(S(n))$ for all $S(n)=o(\log n)$.

Proof. If the weak Goldbach's conjecture is true, then $L_{W G} \notin R E G$ as a consequence of Theorem 2. However, if $L_{W V G} \in N S P A C E(S(n))$ for some $S(n)=o(\log n)$, then $L_{W G} \in$ $R E G$ due to Theorem 6. In this way, the weak Goldbach's conjecture cannot be true under the assumption that $L_{W V G} \in N S P A C E(S(n))$ for some $S(n)=o(\log n)$. Since the weak Goldbach's conjecture is true, then we obtain that $L_{W V G} \notin N \operatorname{SPACE}(S(n))$ for all $S(n)=o(\log n)[15],[16]$.

The checking whether a number is prime can be decided in polynomial time by a deterministic Turing machine [1]. This problem is known as PRIMES [1].

- Theorem 8. PRIMES $\notin N S P A C E(S(n))$ for all $S(n)=o(\log n)$.

Proof. From the Theorem 7, we obtain that $L_{W V G} \notin N S P A C E(S(n))$ for all $S(n)=o(\log n)$. However, the checking of whether the four numbers on the input are odds and proving the equality of the sum's equation can be done in $N S P A C E(o(\log n))$. Certainly, the verification of the odd property could be done in constant space. In addition, the verification of the equality of the sum's equation $2 \times n+1=p+q+r$ can be done in NSPACE $(o(\log n))$. 
Indeed, given four natural numbers $p, q, r$ and $t$ in binary encoding, it is obviously possible to check in $N S P A C E(\log n)$ whether $p+q+r=t$. We need to go through corresponding bits from $p, q, r$ and $t$ starting from least significant bits to most significant bits. So for each $i$ from 1 to $n$, we check if $p, q, r$ and $t$ have compatible/matching bits at position $i$ (i.e. $p_{i}$, $q_{i}, r_{i}$, and $t_{i}$ are compatible). Then, we keep track of any carry bit in constant space and move to index $i+1$. We just need to keep track of $i$ written in binary. If $n$ is the greatest bit length between $p, q, r$ and $t$, then we need $\log n$ bits to keep track of $i$. However, we can keep track of $i$ using $o(\log n)$ space.

The position $i$ is stored using a triple $(a, b, c)$ of binary strings that represent positive integers. In the least significant bit position we use $(1,0,0)$. For a current bit position $i$ in a triple $(a, b, c)$, we move for the new bit position $i+1$ using the rules of the following steps:

1. If $0<a<\left\lfloor\frac{n}{\log n}\right\rfloor$, then the next step $i+1$ into the new bit position is $(a+1, b, c)$,

2. else if $a=\left\lfloor\frac{n}{\log n}\right\rfloor$, then the next step $i+1$ into the new bit position is $(0,0,1)$,

3. else if $a=0$ then:

a. if $c=\lfloor\log n\rfloor$, then the next step $i+1$ into the new bit position is $(a, b+1,1)$ otherwise if $c \neq\lfloor\log n\rfloor$, then the next step $i+1$ into the new bit position is $(a, b, c+1)$.

Every triple $(a, b, c)$ represents the bit position $a \leq\left\lfloor\frac{n}{\log n}\right\rfloor$ when $a>0$ or $\left\lfloor\frac{n}{\log n}\right\rfloor+$ $(\lfloor\log n\rfloor \times b)+c$ when $a=0$. In this way, $b$ and $c$ always comply with $c \leq\lfloor\log n\rfloor$ and $b \leq \frac{n-\left\lfloor\frac{n}{\log n}\right\rfloor}{\lfloor\log n\rfloor}$. Certainly, this is based on the following equation

$$
\left\lfloor\frac{n}{\log n}\right\rfloor+\lfloor\log n\rfloor \times \frac{n-\left\lfloor\frac{n}{\log n}\right\rfloor}{\lfloor\log n\rfloor}=n
$$

However, the bit length of $\lfloor\log n\rfloor$ is bounded by $\log \lfloor\log n\rfloor$. In addition, the bit length of $\left\lfloor\frac{n}{\log n}\right\rfloor$ is bounded by $\log n-\log \log n$. Moreover, the bit length of the integer part of $\frac{n-\left\lfloor\frac{n}{\log n}\right\rfloor}{\lfloor\log n\rfloor}$ is bounded by $\log \left(n-\left\lfloor\frac{n}{\log n}\right\rfloor\right)-\log \lfloor\log n\rfloor$. Since we add the bit length of $c$ in case of $a=0$, then this will be $\log \left(n-\left\lfloor\frac{n}{\log n}\right\rfloor\right)$. In this way, the whole computation is bounded by $\log \left(n-\left\lfloor\frac{n}{\log n}\right\rfloor\right)$ or $\log n-\log \log n$ space. Furthermore, we use a single triple $\left(a^{\prime}, b^{\prime}, c^{\prime}\right)$ to put the head into the bit positions of the binary numbers:

1. if we want to put the head of the tape into the bit position $\left(a^{\prime}, b^{\prime}, c^{\prime}\right)$ inside of a binary string, then we just set the head in the least significant bit position and move to the left while we decrement in 1 the bit position using the same rules that we used for incrementing until we reach the value $(1,0,0)$

2. and after that, if we want to put the head of the tape into the bit position $\left(a^{\prime}, b^{\prime}, c^{\prime}\right)$ inside of another binary string, then from the current position in the head tape, we move to the right while we just increment in 1 the bit position from the value $(1,0,0)$ using the same rules above until the head will stay in the least significant bit position of the current binary string reaching the previous value $\left(a^{\prime}, b^{\prime}, c^{\prime}\right)$

3. and while we doing that, we copy the bits $p_{i}, q_{i}, r_{i}$, and $t_{i}$ to the work tapes from the bit position $i$ that represents $\left(a^{\prime}, b^{\prime}, c^{\prime}\right)$ and do the necessary verification

4. and finally, when we finish all that, then we erase the bits $p_{i}, q_{i}, r_{i}$, and $t_{i}$ and create the next step $i+1$ from the value $\left(a^{\prime}, b^{\prime}, c^{\prime}\right)$ into the new bit position using the same rules above.

However, we know that $\log n-\log \log n=o(\log n)$ and $\log \left(n-\left\lfloor\frac{n}{\log n}\right\rfloor\right)=o(\log n)$ for $n \geq 3$ where the whole computation can be done in a nondeterministic way because of it is indeed deterministic [21]. In addition, the ultimate remaining verification that we need to 
analyze in $L_{W V G}$ is whether $p, q$ and $r$ are primes. Since the other properties can be done in $N S P A C E(o(\log n))$ excluding the primality test and $L_{W V G} \notin N S P A C E(S(n))$ for all $S(n)=$ $o(\log n)$, then we have as unique remaining possibility that PRIMES $\notin N S P A C E(S(n))$ for all $S(n)=o(\log n)$.

- Theorem 9. The strong Goldbach's conjecture is true or this has an infinite number of counterexamples.

Proof. If the strong Goldbach's conjecture is false, then $L_{G} \in R E G$ or $L_{G}$ is non-regular and its complement is infinite, since every finite set is regular and $R E G$ is also closed under complement [21]. However, this implies that the exponentially more succinct version of $L_{G}$, that is $L_{V G}$, should be in $N S P A C E(S(n))$ for some $S(n)=o(\log n)$, because of $R E G=$ $N S P A C E(o(\log \log n))$ and the same algorithm that decides $L_{G}$ in $N S P A C E(o(\log \log n))$ could be easily transformed into a slightly modified algorithm that decides $L_{V G}$ in $N \operatorname{SPACE}(S(n))$ for some $S(n)=o(\log n)$ [18], [12]. Actually, $L_{G}$ could be reduced to $L_{S G}$ in a nondeterministic constant space following the steps of Theorem 6 and $L_{S G}$ is the unary version of $L_{V G}$ due to Lemma 5 . As we mentioned before, the standard space translation between the unary and binary languages actually works for nondeterministic machines with small space [12]. This means that if some unary language belongs to $N S P A C E(S(\log n))$, then the binary version of that language belongs to $N S P A C E(S(n))$ [12]. It is not possible that $L_{V G} \in N S P A C E(S(n))$ for some $S(n)=o(\log n)$, because of PRIMES $\notin N S P A C E(S(n))$ for all $S(n)=o(\log n)$. Certainly, the verification of whether $p$ and $q$ are primes need to be done in order to accept the elements of this language. Consequently, we obtain that $L_{G} \notin R E G$, since it is not possible that $L_{G} \in N \operatorname{SPACE}(o(\log \log n))$ under the result of $L_{V G} \notin N S P A C E(S(n))$ for all $S(n)=o(\log n)$. In this way, we obtain a contradiction just assuming that the strong Goldbach's conjecture is false and $L_{G} \in R E G$. In contraposition, we have the strong Goldbach's conjecture is true or this has an infinite number of counterexamples.

\subsection{Beal's conjecture}

- Definition 10. For a specific choice of exponents $(x, y, z)$ where $x, y, z \in \mathbb{N}$ and $x, y, z \geq 3$, we define the Beal's language $L_{B}$ as follows:

$$
L_{B}=\left\{1^{r} 0^{p} 0^{q}: p, q, r \in \mathbb{N} \wedge p \leq q \wedge r=p+q\right\}
$$

where when $p=1$ then $r$ has not a perfect $z$-root or $r$ has a perfect $z$-root and there are no positive integers $p$ and $q$ such that $r=p+q, p$ has a perfect $x$-root and $q$ has a perfect $y$-root otherwise when $p>1$ then $p, q$ and $r$ are not co-primes and the greatest common divisor between $p, q$ and $r$ has the smallest possible value such that $r$ has a perfect $z$-root, $p$ has a perfect $x$-root and $q$ has a perfect $y$-root.

- Theorem 11. If the Beal's conjecture is true, then the Beal's language $L_{B}$ is non-regular.

Proof. If the Beal's conjecture is true, then the Beal's language $L_{B}$ is equal to the another language $L^{\prime}$ defined as follows:

$$
L^{\prime}=\left\{1^{n} 0^{n}: n \in \mathbb{N} \wedge n \geq 2\right\} .
$$

We can easily prove that $L^{\prime}$ is non-regular using the Pumping lemma for regular languages [23].

- Definition 12. We define the verification Beal's language $L_{V B}$ as follows:

$$
L_{V B}=\left\{(r, p, q) \text { : such that } 1^{r} 0^{p} 0^{q} \in L_{B}\right\} .
$$


- Definition 13. We define the Beal's language with separator $L_{S B}$ as follows:

$$
L_{S B}=\left\{0^{r} \# 0^{p} \# 0^{q}: \text { such that } 1^{r} 0^{p} 0^{q} \in L_{B}\right\}
$$

where \# is the blank symbol.

- Lemma 14. The Beal's language with separator $L_{S B}$ is the unary representation of the verification Beal's language $L_{V B}$.

Proof. This is trivially true from the definition of these languages.

- Theorem 15. $L_{V B} \notin N S P A C E(S(n))$ for all $S(n)=o(\log n)$.

Proof. The complement $c o L_{V B}$ must check the factorization into prime numbers or the greatest common divisor in order to prove that the three numbers are co-primes, since the greatest common divisor can in principle be computed by determining the prime factorizations of the three numbers [14]. Certainly, $c o L_{V B}$ should contain the possible counterexamples of the Beal's conjecture for the chosen exponents $(x, y, z)$ in $c o L_{V B}$. The COMPOSITE problem is the complement of PRIMES language. Indeed, the computation of the greatest common divisor cannot be computed in $N S P A C E(S(n))$ for some $S(n)=o(\log n)$, because of this would imply that the COMPOSITE problem is in $N S P A C E(S(n))$ for some $S(n)=$ $o(\log n)$ as well.

Certainly if this could be true, then we can go from the numbers 2 to $n-1$ and check whether the greatest common divisor with $n$ is not 1 . This could be nondeterministically done on input $n$ just choosing another number lesser than $n$ and greater than 1 , but instead of putting in the work tapes, then this will put with $n$ in the output tape just using constant space into one-way [18]. After that, we use the nondeterministic logarithmic space composition reduction just using the previous output of $n$ and some $2 \leq i \leq n-1$ into a new nondeterministic Turing machine that would compute the greatest common divisor in $N S P A C E(S(n))$ for some $S(n)=o(\log n)$ using $(n, i)$ as input [21]. Since the first Turing machine is in one-way, then the whole process could be done in $N \operatorname{SPACE}(S(n))$ for some $S(n)=o(\log n)$.

However, this would be a contradiction according to Theorem 8 , since PRIMES $N S P A C E(S(n))$ for all $S(n)=o(\log n)$. The reason is because of $N S P A C E(S(n))$ is closed under complement for $S(n) \geq \log n$ [18]. Hence, if PRIMES $\notin N S P A C E(S(n))$ for all $S(n)=o(\log n)$, then $C O M P O S I T E \notin N S P A C E(S(n))$ for all $S(n)=o(\log n)$. In addition, the integer factorization cannot be done in $N S P A C E(S(n))$ for some $S(n)=o(\log n)$, due to this needs to check whether the composition of factors are solely composed of prime numbers. Since $c o L_{V B}$ depends mostly on the factorization into prime numbers or the computation of the greatest common divisor in order to accept its elements, then $\operatorname{coL}_{V B} \notin N S P A C E(S(n))$ for all $S(n)=o(\log n)$. In this way, we obtain that $L_{V B} \notin N S P A C E(S(n))$ for all $S(n)=o(\log n)$ $[18]$.

- Theorem 16. The Beal's conjecture is true.

Proof. If the Beal's conjecture is false, then $L_{B} \in R E G$ or $L_{B}$ is non-regular and its complement is infinite, since every finite set is regular and $R E G$ is also closed under complement [21]. However, this implies that the exponentially more succinct version of $L_{B}$, that is $L_{V B}$, should be in $N S P A C E(S(n))$ for some $S(n)=o(\log n)$, because of $R E G=N S P A C E(o(\log \log n))$ and the same algorithm that decides $L_{B}$ in $N S P A C E(o(\log \log n))$ could be easily transformed into a slightly modified algorithm that decides $L_{V B}$ in $N S P A C E(S(n))$ for some $S(n)=o(\log n)$ [18], [12]. Actually, $L_{B}$ could be reduced to $L_{S B}$ in a nondeterministic 
constant space following the steps of Theorem 6 and $L_{S B}$ is the unary version of $L_{V B}$ due to Lemma 14. As we mentioned before, the standard space translation between the unary and binary languages actually works for nondeterministic machines with small space [12]. This means that if some unary language belongs to $N S P A C E(S(\log n))$, then the binary version of that language belongs to $N S P A C E(S(n))$ [12]. In this way, we obtain that $L_{B} \notin R E G$, since it is not possible that $L_{B} \in N S P A C E(o(\log \log n))$ under the result of $L_{V B} \notin N S P A C E(S(n))$ for all $S(n)=o(\log n)$ as a consequence of Theorem 15. Consequently, we obtain a contradiction just assuming that the Beal's conjecture is false and $L_{B} \in R E G$. In contraposition, we have the Beal's conjecture is true or this has an infinite number of counterexamples both for a specific choice of exponents $(x, y, z)$, since $L_{B}$ uses a specific choice of exponents $(x, y, z)$. The Darmon-Granville theorem uses Faltings's theorem to show that for every specific choice of exponents $(x, y, z)$, there are at most finitely many co-prime solutions for $(A, B, C)[9],[11]$. In conclusion, we obtain that necessarily the Beal's conjecture is true for this specific choice of exponents $(x, y, z)$ as the remaining only option. Since we took arbitrarily the exponents $(x, y, z)$, then the Beal's conjecture will be true for every specific choice of exponents $(x, y, z)$.

\section{Conclusions}

Statistical considerations that focus on the probabilistic distribution of prime numbers present informal evidence in pos of the strong conjecture for sufficiently large integers: The greater the integer, the more ways there are available for that number to be represented as the sum of two other numbers, and the more "likely" it becomes that at least one of these representations consists entirely of primes. In this way, the statement that the strong Goldbach's conjecture has an infinite number of counterexamples is certainly "unlikely". To sum up, this work represents a big step forward in showing the strong Goldbach's conjecture should be really true.

Peter Norvig, Director of Research at Google, have conducted a series of numerical searches for counterexamples to Beal's conjecture. Among his results, he excluded all possible solutions having each of $x, y, z=7$ and each of $A, B, C=250,000$, as well as possible solutions having each of $x, y, z=100$ and each of $A, B, C=10,000$ [20]. We conclude announcing the failure in the prolonged search of counterexamples since the Beal's conjecture is true.

Fermat's Last Theorem established that $A^{n}+B^{n}=C^{n}$ has no solutions for $n>2$ for positive integers $A, B$, and $C$. If any solutions had existed to Fermat's Last Theorem, then by dividing out every common factor, there would also exist solutions with $A, B$, and $C$ co-prime which would mean they do not have a common prime factor [14]. Hence, Fermat's Last Theorem can be seen as a special case of the Beal's conjecture restricted to $x=y=z$ [4].

The Fermat-Catalan conjecture is that $A^{x}+B^{y}=C^{z}$ has only finitely many solutions with $A, B$, and $C$ being positive integers with no common prime factor and $x, y$, and $z$ being positive integers satisfying $\frac{1}{x}+\frac{1}{y}+\frac{1}{z}<1[29]$. Beal's conjecture can be restated as "All Fermat-Catalan conjecture solutions will use 2 as an exponent".

\section{References}

1 Manindra Agrawal, Neeraj Kayal, and Nitin Saxena. PRIMES is in P. Annals of Mathematics, 160(2):781-793, 2004. doi:10.4007/annals.2004.160.781. 
2 Alfred V. Aho and John E. Hopcroft. The Design and Analysis of Computer Algorithms. Pearson Education India, 1974.

3 Sanjeev Arora and Boaz Barak. Computational complexity: a modern approach. Cambridge University Press, 2009.

4 Michael A. Bennett, Imin Chen, Sander R. Dahmen, and Soroosh Yazdani. Generalized Fermat Equations: A Miscellany, June 2014. Available at http://people.math.sfu.ca/ ichen/pub/ BeChDaYa.pdf. Retrieved 21 February 2020.

5 Frits Beukers. The generalized fermat equation, January 2006. Available at http://www . staff.science.uu.nl/ beuke106/Fermatlectures.pdf. Retrieved 21 February 2020.

6 Jing-run Chen. On the Representation of a Large Even Integer as the Sum of a Prime and the Product of Two Primes at Most. Sci. Sinica, 16:157-176, 1973.

7 Thomas H. Cormen, Charles E. Leiserson, Ronald L. Rivest, and Clifford Stein. Introduction to Algorithms. The MIT Press, 3 edition, 2009.

8 Richard Crandall and Carl Pomerance. Prime Numbers: A Computational Perspective. Springer, 2000.

9 Henri Darmon and Andrew Granville. On the equations $z^{m}=F(x, y)$ and $A x^{p}+B y^{q}=C z^{r}$. Bulletin of the London Mathematical Society, 27:513-543, 1995.

10 Leonard Eugene Dickson. History of the Theory of Numbers: Divisibility and Primality, volume 1. New York, Dover, 2005.

11 Noam D. Elkies. The ABC's of Number Theory. The Harvard College Mathematics Review, 1(1), 2007.

12 Viliam Geffert and Dana Pardubská. Unary Coded NP-Complete Languages in ASPACE (log $\log$ n). International Journal of Foundations of Computer Science, 24(07):1167-1182, 2013. doi:10.1007/978-3-642-31653-1_16.

13 Richard K. Guy. Unsolved Problems in Number Theory. New York, Springer-Verlag, 3 edition, 2004.

14 Godfrey Harold Hardy and Edward Maitland Wright. An Introduction to the Theory of Numbers. Oxford University Press, 1979.

15 Harald A. Helfgott. Minor arcs for Goldbach's problem. arXiv preprint arXiv:1205.5252, 2012.

16 Harald A. Helfgott. Major arcs for Goldbach's theorem. arXiv preprint arXiv:1305.2897, 2013.

17 R. Daniel Mauldin. A Generalization of Fermat's Last Theorem: The Beal Conjecture and Prize Problem. Notices of the AMS, 44(11):1436-1439, 1997.

18 Pascal Michel. A survey of space complexity. Theoretical computer science, 101(1):99-132, 1992. doi: 10.1016/0304-3975 (92) 90151-5.

19 Abderrahmane Nitaj. On A Conjecture of Erdos on 3-Powerful Numbers. Bulletin of the London Mathematical Society, 27(4):317-318, 1995.

20 Peter Norvig. Beal's Conjecture: A Search for Counterexamples, October 2015. Available at http://norvig.com/beal.html. Retrieved 21 February 2020.

21 Christos H. Papadimitriou. Computational complexity. Addison-Wesley, 1994.

22 Bjorn Poonen, Edward F. Schaefer, and Michael Stoll. Twists of $x(7)$ and primitive solutions to $x^{2}+y^{3}=z^{7}$. Duke Mathematical Journal, 137:103-158, 2005.

23 Michael O. Rabin and Dana Scott. Finite automata and their decision problems. IBM journal of research and development, 3(2):114-125, 1959. doi:10.1147/rd.32.0114.

24 John C. Shepherdson. The Reduction of Two-Way Automata to One-Way Automata. IBM Journal of Research and Development, 3(2):198-200, 1959. doi:10.1147/rd.32.0198.

25 Samir Siksek and Michael Stoll. The generalised fermat equation $x^{2}+y^{3}=z^{15}$. Archiv der Mathematik, 102:411-421, 2013.

26 Joseph H. Silverman. A Friendly Introduction to Number Theory. Pearson Education, Inc., 4 edition, 2012.

27 Michael Sipser. Introduction to the Theory of Computation, volume 2. Thomson Course Technology Boston, 2006. 
28 Ivan M. Vinogradov. Representation of an Odd Number as a Sum of Three Primes. Comptes Rendus (Doklady) de l'Académie des Sciences de l'USSR, 15:169-172, 1937.

29 David G. Wells. Prime Numbers, The Most Mysterious Figures in Math. John Wiley \& Sons, Inc., 2005.

30 Andrew Wiles. Modular elliptic curves and Fermat's Last Theorem. Annals of Mathematics, 141(3):443-551, 1995. doi:0.2307/2118559. 